•数据论文・

\title{
中国亚热带4个森林动态监测样地无人机可见光遥 感影像数据集
}

张昭臣 ${ }^{1}$, 胡健波 ${ }^{2}$, 杨庆松 ${ }^{1}$, 练琚愉 ${ }^{3}$, 李步杭 ${ }^{4}$, 王希华 ${ }^{1}$, 叶万辉 ${ }^{3}$, 张健 (1) ${ }^{*}$

1. 华东师范大学生态与环境科学学院, 浙江天童森林生态系统国家野外科学观测研究站, 上海 $200241 ; 2$. 交通运输部天津水利水运工程研 究所, 天津 300456; 3. 中国科学院华南植物园退化生态系统植被恢复与管理重点实验室, 广州 510650; 4. 中山大学有害生物控制与资源利 用国家重点实验室, 中山大学生命科学学院, 广州 510275

摘要: 常绿阔叶林是我国亚热带地区的地带性植被类型。由于亚热带森林植物群落垂直结构复杂、林冠郁闭度高, 对常绿阔 叶林冠层的研究尚缺乏高质量的监测数据。本数据集包含浙江天童山、浙江百山祖、广东车八岭、广东鼎湖山 4 个大于 20 ha 的森林动态监测样地2014年8月或2016年9月采集的无人机可见光遥感影像。本数据集是通过将无人机影像、地面控制点和地 面调查数据相结合而获得的。每个样地的数据集包括 4 个文件: $5 \mathrm{~cm}$ 空间分辨率的正射影像图和数字表面模型、 $1 \mathrm{~m}$ 空间分 辨率的森林冠层高度数据和正射影像质量报告。本数据集可为常绿阔叶林的林冠生态学、生物多样性、生态系统功能等研究 提供数据支撑。

关键词: 无人机遥感; 近地面遥感; 常绿阔叶林; 森林动态监测样地; 林冠生态学

数据库(集)基本信息简介

\begin{tabular}{ll}
\hline 数据库(集)名称 & 中国亚热带4个森林动态监测样地无人机可见光遥感影像数据集 \\
作者 & 张昭臣, 胡健波, 杨庆松, 练琚愉, 李步杭, 王希华, 叶万辉, 张健 \\
通讯作者 & 张健(jzhang@des.ecnu.edu.cn) \\
时间范围 & 2014 年8月或2016年9月 \\
地理区域 & $29^{\circ} 48^{\prime} \mathrm{N}, 121^{\circ} 47^{\prime} \mathrm{E}$, 浙江省天童山国家森林公园; $27^{\circ} 45^{\prime} \mathrm{N}, 119^{\circ} 12^{\prime} \mathrm{E}$, 浙江省凤阳山-百山祖国家级自然保护区; \\
& $24^{\circ} 43^{\prime} \mathrm{N}, 114^{\circ} 15^{\prime} \mathrm{E}$, 广东省车八岭国家级自然保护区; $23^{\circ} 10^{\prime} \mathrm{N}, 112^{\circ} 32^{\prime} \mathrm{E}$, 广东省鼎湖山国家级自然保护区 \\
空间分辨率 & 正射影像与数字表面模型: cm; 林冠层高度图: $1 \mathrm{~m}$ \\
文件大小 & $5.48 \mathrm{G}$ \\
数据格式 & GeoTiff; CSV \\
数据链接 & http://dataopen.info/article/212 \\
& http://doi.org/10.24899/do.202107002 \\
数据库(集)组成 & https://www.biodiversity-science.net/fileup/1005-0094/DATA/2021299.zip \\
\end{tabular}

张昭臣, 胡健波, 杨庆松, 练琚愉, 李步杭, 王希华, 叶万辉, 张健 (2021) 中国亚热带4个森林动态监测样地无人机可见光遥感影像数据集. 生物多样 性, 29, 1181-1185. doi: 10.17520/biods.2021299.

Zhang ZC, Hu JB, Yang QS, Lian JY, Li BH, Wang XH, Ye WH, Zhang J (2021) An unmanned aerial vehicle visible light remote sensing image dataset of four forest dynamic plots in subtropical China. Biodiversity Science, 29, 1181-1185. doi: 10.17520/biods.2021299.

\section{An unmanned aerial vehicle visible light remote sensing image dataset of four forest dynamic plots in subtropical China}

Zhaochen Zhang ${ }^{1}$, Jianbo $\mathrm{Hu}^{2}$, Qingsong Yang ${ }^{1}$, Juyu Lian ${ }^{3}$, Buhang $\mathrm{Li}^{4}$, Xihua Wang ${ }^{1}$, Wanhui Ye ${ }^{3}$, Jian Zhang ${ }^{\left(11^{*}\right.}$

1 Zhejiang Tiantong Forest Ecosystem National Observation and Research Station, School of Ecological and Environmental Sciences, East China Normal University, Shanghai 200241

2 Tianjin Research Institute of Water Transport Engineering, Ministry of Transport, Tianjin 300456

收稿日期: 2021-07-27; 接受日期: 2021-09-05

基金项目: 国家自然科学基金(32071538; 31670439)

* 通讯作者 Author for correspondence. E-mail: jzhang@des.ecnu.edu.cn 
3 Key Laboratory of Vegetation Restoration and Management of Degraded Ecosystems, South China Botanical Garden, Chinese Academy of Sciences, Guangzhou 510650

4 State Key Laboratory of Biocontrol, School of Life Sciences, Sun Yat-sen University, Guangzhou 510275

\section{ABSTRACT}

The evergreen broad-leaved forest is a zonal vegetation type in the subtropical regions of China. However, due to complex vertical structure and relatively dense canopy of subtropical forests, we are lack of reliable data in current canopy studies of this forest. Here we present a large dataset of visible light remote sensing images of four large-scale (> 20 ha) forest dynamic plots in Tiantongshan and Baishanzu of Zhejiang, and Dinghushan and Chebaling of Guangdong. These data were collected by unmanned aerial vehicle (UAV) equipped with visible light camera in August of 2014 or September of 2016. By combining the UAV data with ground survey data, we generated four data files for each plot: orthoimages and surface digital models with spatial resolution of $\sim 5 \mathrm{~cm}$, forest canopy height data with spatial resolution of $1 \mathrm{~m}$, and data quality reports of the orthoimages. This dataset of evergreen broad-leaved forest provides significant supports for the studies in forest canopy ecology, biodiversity and ecosystem function.

Key words: UAV-based remote sensing; near-surface remote sensing; evergreen broad-leaved forest; forest dynamics plot; forest canopy ecology.

\section{Database/Dataset Profile}

\begin{tabular}{|c|c|}
\hline Title & $\begin{array}{l}\text { An unmanned aerial vehicle visible light remote sensing image dataset of } 4 \text { subtropical forest dynamic monitoring plots in } \\
\text { China }\end{array}$ \\
\hline Authors & Zhaochen Zhang, Jianbo Hu, Qingsong Yang, Juyu Lian, Buhang Li, Xihua Wang, Wanhui Ye, Jian Zhang \\
\hline Corresponding author & Jian Zhang (jzhang@des.ecnu.edu.cn) \\
\hline Time range & August of 2014 or September of 2016 \\
\hline Geographical scope & $\begin{array}{l}29^{\circ} 48^{\prime} \mathrm{N}, 121^{\circ} 47^{\prime} \mathrm{E} \text {, Tiantong National Forest Park, Zhejiang Province; } 27^{\circ} 45 \mathrm{~N}, 119^{\circ} 12^{\prime} \mathrm{E} \text {, Fengyangshan- Baishanzu } \\
\text { National Nature Reserve, Zhejiang Province; } 24^{\circ} 4 \mathrm{~S}, 114^{\circ} 15^{\prime} \mathrm{E} \text {, Chebaling National Nature Reserve, Guangdong } \\
\text { Province; } 23^{\circ} 10^{\prime} \mathrm{N}, 112^{\circ} 32^{\prime} \text { E, Dinghushan National Nature Reserve, Guangdong Province }\end{array}$ \\
\hline Spatial resolution & Orthoimages and the digital elevation model: $\sim 5 \mathrm{~cm}$, UAV-driven canopy height map: $1 \mathrm{~m}$ \\
\hline File size & $5.48 \mathrm{G}$ \\
\hline Data format & GeoTiff; CSV \\
\hline Data link & $\begin{array}{l}\text { http://dataopen.info/article/212 } \\
\text { http://doi.org/10.24899/do.202107002 } \\
\text { https://www.biodiversity-science.net/fileup/1005-0094/DATA/2021299.zip }\end{array}$ \\
\hline $\begin{array}{l}\text { Database/Dataset } \\
\text { composition }\end{array}$ & $\begin{array}{l}\text { The dataset consists of } 16 \text { data files in total. For each plot, it includes orthoimages, digital surface model, canopy height } \\
\text { maps, and data quality reports of the orthoimages. }\end{array}$ \\
\hline
\end{tabular}

常绿榈叶林是我国亚热带地区的地带性植被 类型(宋永昌, 2013)。由于其垂直结构复杂、林冠郁 闭度较高, 传统的基于地面的冠层结构测量方法成 本高、覆盖范围小、获得数据精度较差(Zhang et al, 2016a)。尽管一些高分辨率的卫星影像已被用来分 析森林冠层结构, 但由于获取图像周期长、实时性 差等限制, 使其很难应用到局域尺度的群落生态学 研究中。近几年来, 基于无人机 (unmanned aerial vehicle, UAV)为载体的近地面遥感技术的快速发展 使得获取实时的高精度遥感影像数据成为可能 (Anderson \& Gaston, 2013; 胡健波和张健, 2018)。

本数据集是我国东部地区 4 个 $20 \mathrm{ha}$ 以上的亚热 带常绿润叶林森林动态监测样地(浙江天童山20 ha 样地、浙江百山祖 25 ha样地、广东车八岭 $25 \mathrm{ha}$ 样地 和广东鼎湖山 $20 \mathrm{ha}$ 样地)的无人机可见光遥感影像
(空间分辨率约 $5 \mathrm{~cm}$ ) 以及林冠层高度数据(空间分 辨率约 $1 \mathrm{~m}$ )。研究人员可利用样地内的树木空间分 布、地形等信息, 结合生态学模型和空间统计学等 方法与技术, 构建各样地的高分辨率三维冠层结构 数据库, 探讨群落尺度上森林垂直方向上的空间异 质性，分析局域或区域尺度上森林冠层结构对森林 生物多样性的影响(Zhang et al, 2016a, b)。这些数据 可以为常绿阔叶林的生物多样性保护、自然保护区 管理、生态系统功能评估等提供科学依据。

\section{数据采集和处理方法}

\section{1 数据采集}

本文中采用的无人机型号为MD4-1000四旋翼 无人机(表1), 该系统主要由无人机飞行平台、飞行 控制系统、遥感系统、作业软件和影像处理软件系 
统、无线电遥控系统组成, 采用的相机为 Sony NEX-5R。其主要性能指标如下: 机身重 $2.65 \mathrm{~kg}$, 巡 航空速为 $12 \mathrm{~m} / \mathrm{s}, 1 \mathrm{~kg}$ 标准载荷且无风情况下续航 时间约30 min。

无人机遥感影像的采集主要包括航拍前期准 备和航拍数据获取两个阶段。前期准备阶段主要包 括: (1)根据研究样地的空间信息和高程数据选择起 降场地和航迹规划(图1), 航迹规划使用无人机自带 的地面控制软件mdCockpit完成; (2)在样地及周边 选取5-10个地面控制点, 采用Trimble RTK (RealTime Kinematic)在1 m精度下测量。地面控制点选择 在样地内或样地附近相对比较开阔的地方(如林窗、 小的道路等)。航拍数据获取阶段需注意: (1)在适航 条件下, 对无人机进行飞行条件测试以及相应的飞 行指令传输等; (2)执行飞行时, 飞行高度始终设置 为距地约 $200 \mathrm{~m}$, 航向重叠率约为70\%, 旁向重叠率 为 $60 \%$ 以上; (3)实时监控，完成航飞拍摄。

研究团队分别于 2016年9月7日完成浙江天童 山样地、2016年9月9日完成浙江百山祖样地、2016 年9月 13 日完成广东车八岭样地和 2014 年 8 月 25 日 完成广东鼎湖山样地的无人机遥感数据采集工作。 这 4 个样地的地面植物多样性调查都是按照CTFSForestGEO (the Smithsonian Center for Tropical Forest Science-Forest Global Earth Observatory)的 标准, 对每一个胸径 $\geq 1 \mathrm{~cm}$ 的植物进行定位、挂牌和 胸径测量, 并鉴定到物种。样地的高程数据是采用 全站仪准确测量每个 $20 \mathrm{~m} \times 20 \mathrm{~m}$ 样方的顶点而获 得(表2)。

\section{2 数据处理方法}

使用Pix4D mapper软件进行图像处理。通过软
表1 MD4-1000四旋翼无人机的相机型号、设置和照片参数 Table 1 The camera type, settings and photograph parameters equipped with MD-4 1000 quadrotor UAV (unmanned aerial vehicle)

\begin{tabular}{ll}
\hline 相机设置 Camera setting & 相关参数 Parameters \\
\hline 相机型号 Camera model & SONY NEX-5R \\
镜头 Camera lens & $16-50 \mathrm{~mm}$ \\
光圈值 The aperture & $\mathrm{f} / 2.8-\mathrm{f} / 5.6$ \\
曝光时间 Exposure time & $1 / 1000$ \\
ISO 速度 ISO speed & ISO-400 \\
焦距 Focal length & $35 \mathrm{~mm}$ \\
最大光圈 Maximum aperture & 1.69 \\
有效像素 Effective pixels/megapixels & 16.1 \\
照片分辨率 Image resolution & $4912 \times 3264$ \\
图片格式 Image format & JPEG \\
\hline
\end{tabular}

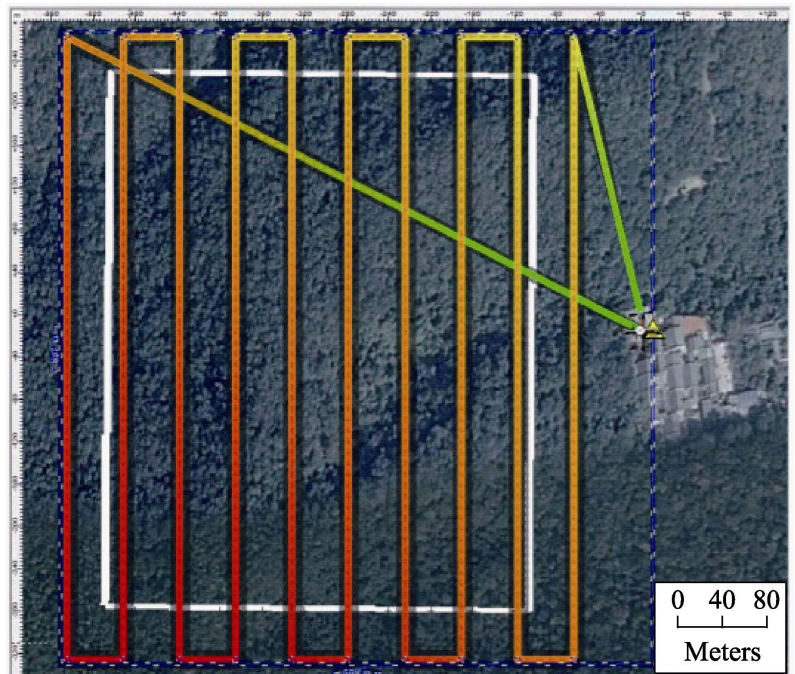

图1 无人机在野外调查时的飞行航线设计示例图

Fig. 1 An example of UAV (unmanned aerial vehicle) mission path

表2 四个亚热带常绿阔叶林森林动态监测样地的基本特征

Table 2 Descriptive statistics for four stem-mapped forest dynamics plots in subtropical regions in China

\begin{tabular}{|c|c|c|c|c|c|c|c|c|}
\hline $\begin{array}{l}\text { 样地名称 } \\
\text { Site }\end{array}$ & $\begin{array}{l}\text { 经纬度 } \\
\text { Location }\end{array}$ & $\begin{array}{l}\text { 年均温 } \\
\text { Mean annual } \\
\text { temperature } \\
\left({ }^{\circ} \mathrm{C}\right)\end{array}$ & $\begin{array}{l}\text { 年降雨 } \\
\text { Annual } \\
\text { precipitation } \\
(\mathrm{mm})\end{array}$ & $\begin{array}{l}\text { 海拔 } \\
\text { Elevation range } \\
(\mathrm{m})\end{array}$ & $\begin{array}{l}\text { 坡度 } \\
\text { Slope } \\
\left({ }^{\circ}\right)\end{array}$ & $\begin{array}{l}\text { 样地大小 } \\
\text { Area } \\
\text { (ha) }\end{array}$ & $\begin{array}{l}\text { 物种数 } \\
\text { Species richness } \\
(\mathrm{DBH} \geq 1 \mathrm{~cm})\end{array}$ & $\begin{array}{l}\text { 总个体数(密度) } \\
\text { Total abundance of } \\
\text { live trees (Density) }\end{array}$ \\
\hline $\begin{array}{l}\text { 浙江天童山 } \\
\text { Tiantongshan, Zhejiang }\end{array}$ & $\begin{array}{l}29^{\circ} 48^{\prime} \mathrm{N} \\
121^{\circ} 47^{\prime} \mathrm{E}\end{array}$ & 16.2 & 1,735 & $304-603$ & $13-50$ & 20 & 152 & 94,603 (4,730/ha) \\
\hline $\begin{array}{l}\text { 浙江百山祖 } \\
\text { Baishanzu, Zhejiang }\end{array}$ & $\begin{array}{l}27^{\circ} 45^{\prime} \mathrm{N} \\
119^{\circ} 13^{\prime} \mathrm{E}\end{array}$ & 12.8 & 2,342 & $1,407-1,646$ & $20-35$ & 25 & 155 & 224,483 (8,979/ha) \\
\hline $\begin{array}{l}\text { 广东车八岭 } \\
\text { Chebaling, Guangdong }\end{array}$ & $\begin{array}{l}24^{\circ} 43^{\prime} \mathrm{N} \\
114^{\circ} 15^{\prime} \mathrm{E}\end{array}$ & 18.1 & 1,900 & $431-545$ & $4-53$ & 20 & 223 & 86,518 (4,316/ha) \\
\hline $\begin{array}{l}\text { 广东鼎湖山 } \\
\text { Dinghushan, Guangdong }\end{array}$ & $\begin{array}{l}23^{\circ} 10^{\prime} \mathrm{N} \\
112^{\circ} 31^{\prime} \mathrm{E}\end{array}$ & 20.9 & 1,985 & $230-470$ & $30-50$ & 20 & 195 & 80,566 (4,028/ha) \\
\hline
\end{tabular}


件块拼检查是否能够形成一张整图, 并检查是否存 在漏拍或俯仰、侧倾过大导致的重叠率过低的现象, 如有则重拍或补拍, 并通过软件自带匀光处理功能 对图像进行匀光处理, 进而生成正射影像图。

天童山、百山祖、车八岭和鼎湖山 4 个样地所 采集的有效照片数量分别为 303 张、289张、228张 和322张。基于无人机采集的航拍图像, 选取地面控 制点对点云进行地理校正。每个控制点的坐标使用 Trimble Geo7X RTK在小于1 m精度下测量。由于各 个保护区样地附近的植被覆盖的差异以及样地地 形复杂程度的差异(表2), 各样地的地面控制点也存 在差异。四个样地地面控制点的数量分别为: 天童 山 16 个, 百山祖 6 个, 车八岭 6 个, 鼎湖山 42 个。

结合地面调查所获得的样地高程数据, 通过比 较数字化坐标和已知的地面坐标来评估空间精度, 最后生成空间分辨率约为 $5 \mathrm{~cm}$ 的数字表面模型 (Digital Surface Model, DSM) 和分辨率为 $1 \mathrm{~m}$ 的冠层 高度模型(Canopy Height Model, CHM)。所有图像采 集和图像处理都使用WGS84坐标系统。我们认为 CHM仅在大于 1 m或更高的范围内才有生态学意义, 同时考虑到地面高程数据的精度而将其数据精度确 定为 $1 \mathrm{~m}$ 。

\section{2 数据描述}

本数据集包含以下文件: (1) 4份样地航拍正射 影像文件; (2) 4份样地DSM文件; (3) 4份1 m精度的 样地林冠高度数据文件(示例见图2); (4) 4份样地质 量报告文件。

\section{3 数据质量控制和评估}

本数据集来源于 4 个亚热带森林动态监测样地 的航拍, 数据获取的整个过程均进行质量把控。首 先, 4 个森林监测样地均采用统一标准进行调查, 所 有样地的无人机遥感数据均由同一位研究人员严 格按照航拍流程进行采集; 其次, 为确保数据的完 整性, 在航拍过程中始终保证无人机在距地面约 $200 \mathrm{~m}$ 的高度飞行, 实际拍摄面积为样地大小的两 倍且航拍照片平均重叠约70\%; 最后, 为降低人为 干扰误差, 后期数据处理阶段的航拍图像拼接、几 何校正等由同一位研究人员完成。数据质量评估的 其他信息详见数据集中各样地质量报告文件。

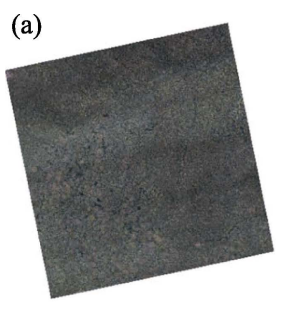

(c)

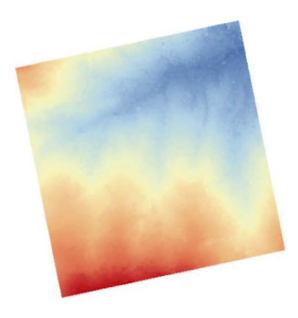

(b)

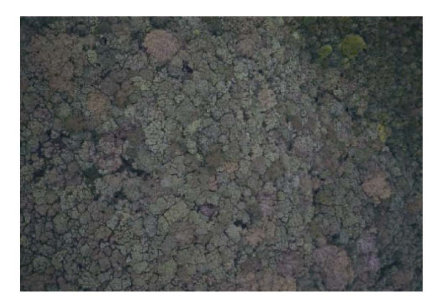

(d)

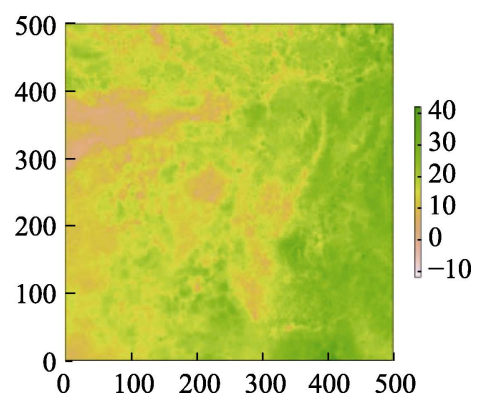

图2 无人机可见光遥感数据示例。(a)正射影像图; (b)无人 机拍摄的原始图片; (c)数字表面模型图; (d)林冠层高度图。

Fig. 2 One example of UAV (unmanned aerial vehicle) visible light remote sensing data. (a) Orthoimages; (b) One of the raw pictures taken by UAV; (c) The map of the digital surface model; and (d) The map of canopy height.

\section{4 数据使用方法和建议}

本数据使用时请注意以下潜在的问题: (1)由于 无人机影像数据与地面调查数据并不能完全匹配, 本数据的空间匹配精度尚存在限制, 研究者应根据 研究问题所对应的空间尺度来确定本数据是否可 以用于自己的研究工作; (2)由于 4 个样地的地面高 程数据是用全站仪在 $20 \mathrm{~m}$ 间隔逐一测量的, 不能获 取每个 $20 \mathrm{~m} \times 20 \mathrm{~m}$ 小样方内的地形变异, 因此本数 据中的DSM数据的精度也存在一定限制, 但足以满 足很多群落生态学研究的需求; (3)本数据为各个样 地的无人机单次调查数据, 采集时间也不完全一 致。尽管对于亚热带成熟森林来说, 在相对稳定的 环境下, 在短期内林冠结构不会有大的变异, 但一 些样地仍会受到台风、风雪灾害等极端天气的影响, 因此在使用本数据时还要注意本数据采集期间的 环境条件是否与研究者使用的其他数据匹配, 以免 在有光时相的分析中失去可比性。

\section{作者分工}

张昭臣主要承担无人机遥感数据收集与论文撰写; 胡健 波主要承担无人机遥感数据收集与数据处理; 杨庆松和王希 华主要承担浙江天童山, 练琚愉和叶万辉主要承担广东鼎湖 山, 李步杭主要承担浙江百山祖和广东车八岭样地地面调查 
数据收集; 张健主要承担研究方案设计与论文撰写。

\section{ORCID}

张健 (D) https://orcid.org/ 0000-0003-0589-6267

\section{参考文献}

Anderson K, Gaston KJ (2013) Lightweight unmanned aerial vehicles will revolutionize spatial ecology. Frontiers in Ecology and the Environment, 11, 138-146.

Hu JB, Zhang J (2018) Unmanned Aerial Vehicle remote sensing in ecology: Advances and prospects. Acta Ecologica Sinica, 38, 20-30. (in Chinese with English abstract) [ 胡健 波, 张健 (2018) 无人机遥感在生态学中的应用进展. 生 态学报, 38, 20-30.]
Song YC (2013) Evergreen Broad-leaved Forests in China: Classification, Ecology, and Conservation. Science Press, Beijing. (in Chinese) [宋永昌 (2013) 中国常绿阔叶林: 分 类・生态・保育. 科学出版社, 北京.]

Zhang J, Hu JB, Lian JY, Fan ZJ, Ouyang XJ, Ye WH (2016a) Seeing the forest from drones: Testing the potential of lightweight drones as a tool for long-term forest monitoring. Biological Conservation, 198, 60-69.

Zhang J, Nielsen SE, Mao LF, Chen SB, Svenning JC (2016b) Regional and historical factors supplement current climate in shaping global forest canopy height. Journal of Ecology, 104, 469-478.

(责任编委：郭庆华 责任编辑：周玉荣) 